\title{
Monitoring and control system of discomfort in disability, bed rest people and surgical patients
}

\author{
M.A.F. Carvalho, a , F.B.N. Ferreira ${ }^{2, b}$, H. Carvalho, ${ }^{3, c}$, J.G. Rocha ${ }^{4, d}$, \\ L.B. Martins ${ }^{5, e}$ and J.A. Santos ${ }^{6, f}$ \\ ${ }^{1,2,3}$ Department of Textile Engineering, University of Minho, Campus de Azurem, \\ 4800-058 Guimaraes, Portugal \\ ${ }^{4}$ Department of Industrial Electronics, University of Minho, Campus de Azurem, \\ 4800-058 Guimaraes, Portugal \\ ${ }^{5}$ Department of Mechanical Engineering, University of Minho, Campus de Azurem, \\ 4800-058 Guimaraes, Portugal \\ ${ }^{6}$ Institute of Education and Psychology, University of Minho, Campus de Gualtar, \\ 4700-056 Braga, Portugal
}

amigcar@det.uminho.pt, 'bnunes@det.uminho.pt, chelder@det.uminho.pt, 'gerardo@dei.uminho.pt, elmartins@dem.uminho.pt, fjorge.a.santos@iep.uminho.pt

Keywords: Health - Prevention of pressure ulcers; Comfort-discomfort; Monitoring and Control; Equality of opportunities - Quality of life

Abstract. Project aims to develop a system that allows to provide to the People with Special Needs (PSN) a relief to the level of the sensitive perception of discomfort, assuring greater independence, welfare, quality of life, prevention of illnesses/wounds, through the development of textile and polymers applications (cushions, mattresses and mattresses overlays) with functions of monitoring and control of pressure in the body's areas in contact with the support surfaces.

In this group of PSN will be enclosed the people with serious motor limitations conditioning their mobility/deambulation, such as bed rest people, patients under effect of sedatives or anaesthesia during long surgeries (intra and post operative), patients and users in general of wheelchairs. These people have, for the most part of the cases, a commitment of sensitivity in the body's areas in contact with the support surfaces, or its motor capacity does not allow them to move regularly of position autonomously, as it would do a healthy person unconsciously. Then, insufficient sanguineous irrigation occurs as result of pressure exceeding too long the tissue capillary pressure depriving tissues of oxygen and essential nutrients, owing to ischemia and hypoxia, which then causes the pressure ulcers (PU) development.

\section{Introduction}

People with serious motor limitations have, in most of the cases, a decrease of sensitivity in the body's areas in contact with the support surfaces. Their limited motor capacity does not allow them to regularly change position autonomously, as a healthy person would do unconsciously. Consequently, insufficient sanguineous irrigation occurs as result of pressure exceeding the tissue capillary pressure for a long time, depriving tissues of oxygen and essential nutrients, owing to ischemia and hypoxia, which then causes the development of pressure ulcers.

The main goal of this project is to investigate the impact of extrinsic factors that contribute to the development of pressure ulcers, to identify critical pressure values in different anatomic points of risk and in addition to assess the impact of a specially designed support surface in preventing that the critical pressure values are reached.

Through the incorporation of sensors, one intends to identify ranges of values of pressure, humidity, temperature and exposure times) corresponding to levels of comfort-discomfort and establish 
relationships between its magnitude and duration and the motor actions, as taken by healthy users. These values will be used to define a protocol of sensor data acquisition versus warnings and actions (displayed on a monitor, through sound, or sending messages to a mobile phone) and/or to an intelligent automatic actuation system that will produce the same results that a healthy user achieves with changes in posture.

The system is intended to enable the inversion of the trend of evolution of these values when critical limits are reached, acting automatically on the position where risk conditions are being identified by the sensing surfaces.

This project is an innovative multidisciplinary undertaking, combining insights of textile, polymers, electronics, mechanics and psychological studies to develop support surface systems able to acquire, record and evaluate body pressure, humidity and temperature, and react at limit values of discomfort with warnings and/or with surface actuation.

\section{State of the art}

Pressure ulcers, also known as decubitus ulcers, pressure sores (or bedsores), are areas of localized damage to the skin and underlying tissue (graduated in stages I to IV) [1,2]. Trauma, burns, spinal cord injury, neurological deficits, peripheral vascular disease, stroke and fecal incontinence emerge as risk factors for pressure ulcers $[3,4]$.

Despite advances in medicine, pressure ulcers remain a major cause of morbidity and mortality.

The common sites of pressure ulcers include the ischium (28\%), the sacrum $(17-27 \%)$, the trochanter $(12-19 \%)$, and the heel $(9-18 \%)$ [1,5,6]. The geriatric and pediatric patients develop frequently ulcers in the occiput because they spend amounts of time lying supine. Pressure ulcers are costly in financial and human terms $[1,7,8]$.

Relieving the pressure in the critical skin point may prevent the development of the ulcer [9].

There are several proposals on the market advertising good results in prevention of pressure ulcers. Their costs are very high and effectiveness is far from satisfying the specialists.

None of the solutions available in the market provide permanent monitoring of the pressure exceeding the comfort limit. Humidity and temperature values have never been measured in association with this subject.

Several critical questions still remain unanswered: How much pressure and/or how long does it take to produce predictable ulceration? To what extent other extrinsic local factors, like temperature or humidity, contribute to skin damage? Is intermittent pressure relief effective in preventing critical pressure? Which characteristics should a support surface present to assure optimal skin conditions?

\section{Objectives of the current project}

Considering the above exposition, we can say this multidisciplinary project pursues several main objectives, all of them related to the prevention of pressure ulcers.

The first objective is the development of a low-cost monitoring system that can be easily integrated into a mattress or cushion, providing continuous measurement of pressure, humidity and temperature.

The second objective is the definition of the control limits of comfort-discomfort (pressure, humidity and temperature) from healthy users induced by static postures. These will be used to develop the warning system. 
The third main objective is the development of the control system. A mechanical actuation system that can compensate the critical factors determined by the monitoring system.

Finally, with the collaboration with HSM (São Marcos Hospital) and APD (Portuguese Association of Disabled People), validate the performance of developed system in clinical environment.

\section{Overview of the system}

The system is based on an array of capacitive sensors arranged in a matrix form (as described in the next section). A custom-designed, signal conditioning and acquisition board, controlled by a 8751 microcontroller, connects to a PC via the microcontroller's serial interface. A program developed in Labview reads, scales and displays the data.

Sensor design. Although, the first objective is the development of a low-cost monitoring system, this project will use existing commercial systems to compare results and guarantee that second and third objectives can be achieved.

The first pressure sensor used described in this paper is based on a parallel plate capacitor with polymeric foam between the plates.

The curve obtained in the relationship between pressure and deformation for the polymeric foam used in the sensor array, according to ASTM D 1621, can generally be very well approximated by a polynomial function. In the low-pressure range, the foam exhibits a quite irregular behavior. Considering that this is the least critical range in terms of discomfort behavior, the resulting measurement error can be neglected and the calibration curve may be approximated by a polynomial function.

Fig. 1 shows a simple circuit for capacitance measurement. The current $\mathrm{i}$ is given by $\mathrm{i}=\mathrm{v} \omega \mathrm{C}$, with $\mathrm{v}$ being the RMS value of applied voltage, $\omega$ the angular frequency of the voltage and $C$ the capacitance. As $v$ and $\omega=2 \pi f$ are constant, the value of $i$ is proportional to the capacitance $C$.

Despite the simplicity of the proposed circuit there is a major drawback: the influence of the parasitic capacitances between the wires that connect the components. The same circuit is shown in Fig. 2, now considering the parasitic capacitances. In this figure, $\mathrm{C}_{\mathrm{P} 1}$ represents the parasitic capacitance between the wire that connects the sensor to the voltage source and the ground; $\mathrm{C}_{\mathrm{P} 2}$ represents the parasitic capacitance between the wire that connects the sensor to the ammeter and the ground and $\mathrm{C}_{\mathrm{P} 3}$ represents the parasitic capacitance between the wire that connects the sensor to the voltage source and the wire that connects the sensor to the ammeter.

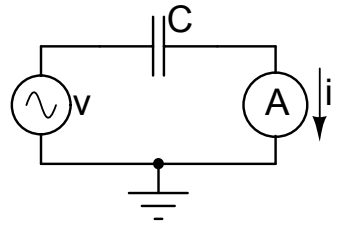

Fig. 1 - Simple circuit for capacitance measurement: the current $i$ is given by $i=v \omega C$. As $v$ and $\omega=2 \pi f$ are constant, the value of $i$ is proportional to the capacitance $\mathrm{C}$.

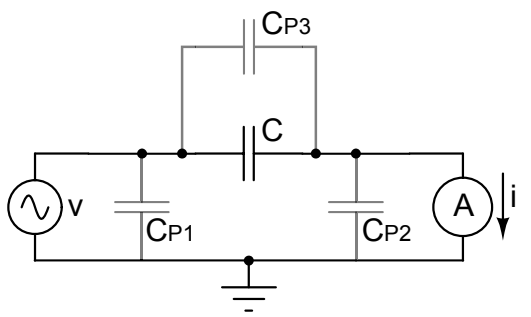

Fig. 2 - Circuit of Fig. 2 with the parasitic capacitances between the wires represented.

It is important to note that the parasitic capacitance $\mathrm{C}_{\mathrm{P} 1}$ is placed parallel to the voltage source, so its value does not influence the measurement. On the other hand, $\mathrm{C}_{\mathrm{P} 2}$ is in parallel with the ammeter, which has very low impedance, so it is short-circuited and it also does not influence the measurement. The main problem is $\mathrm{C}_{\mathrm{P} 3}$, which is in parallel with the sensor capacitance and affects its measurement. In order to solve this problem, either the wire that connects the voltage source to 
the sensor, or the wire that connects the sensor to the ammeter (or both) must be shielded, with its shield connected to the ground. In this case, either the $C_{P 1}$ value or $C_{P 2}$ value (or both values) increase, but, as seen before, they do not influence the measurements.

In the present prototype, an array of $8 \times 8$ sensors was used, as it shown in Fig. 3. The bottom electrode is connected to the voltage source by means of a shielded wire. The top electrodes are connected to the readout circuit, which will be described in the next section.

Fig. 4 shows a picture of the sensor array before its placement inside the cushion.

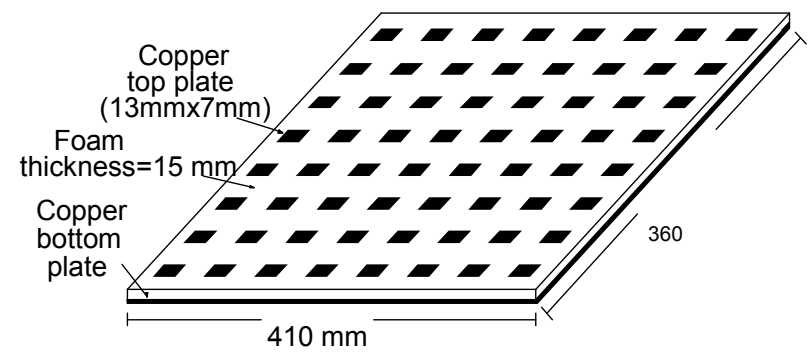

Fig. 3 - Array of $8 \times 8$ capacitive sensors. The bottom electrode consists of a copper sheet with $410 \mathrm{~mm} \times 360 \mathrm{~mm}$; between the electrodes, a polymeric foam layer with $15 \mathrm{~mm}$ thickness was placed; the top electrodes consist in an array of $8 \times 8$ copper plates with $13 \mathrm{~mm} \times 7 \mathrm{~mm}$.

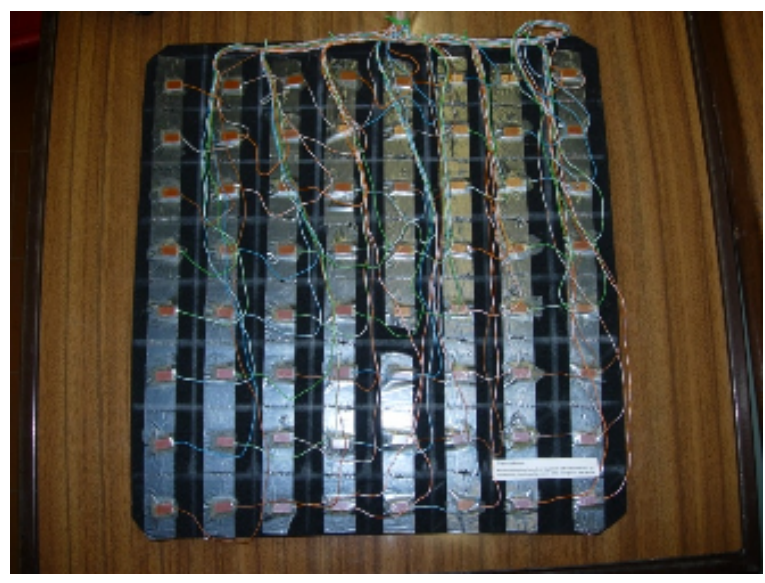

Fig. 4 - Sensor array.

\section{Circuit design}

Voltage source. The voltage source, a Wien Bridge oscillator, working at the frequency of $40 \mathrm{kHz}$, has been used. The output voltage is $10 \mathrm{~V}_{\mathrm{pp}}$. Fig. 5 shows the schematic diagram of the implemented circuit.

Readout circuit. In the sensor, the output current is proportional to the sensor's capacitance. When pressure is applied to the sensor, capacitance varies inversely proportional to the distance between the capacitor plates and thus proportionally to the applied pressure level.

In order to read the pressure level, it is necessary to convert the current supplied by the sensor into a voltage, since the data acquisition board works with voltages at its input. For that, it was necessary to implement the circuit of Fig. 6.

The output of the circuit of Fig. 7, an AC voltage, most be rectified in order to be read by the data acquisition system. As we are dealing with small signals, it was be necessary to implement the circuit of Fig. 7 a precision rectifier for small input voltages.

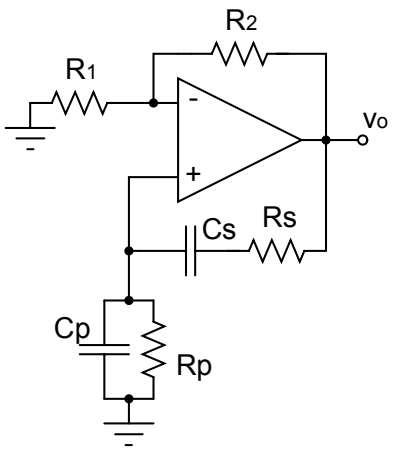

Fig. 5 - Voltage source based on a Wien bridge oscillator.

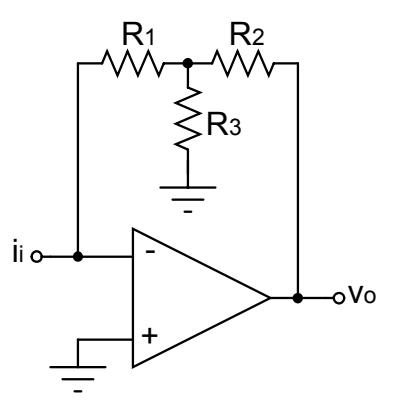

Fig. 6 - Current-voltage converter.

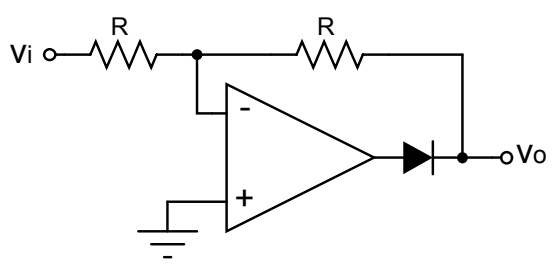

Fig. 7 - Precision full-wave rectifier. 
Finally, as the movement of the patients occurs at a low frequency, a first-order low-pass filter is used. The main goal of this filter is to eliminate the $80 \mathrm{kHz}$ frequency that comes from the full wave rectifier.

\section{Software design}

The acquisition and display software is being developed in Labview. At the current stage of this work, it is a very simple program providing:

- Serial communication with the data acquisition board, reading the acquired values from the board;

- Scaling of the binary values according to pre-defined calibration curves;

- Display of the values on a 2D graph in which the pressure values are mapped to a color scale.

Additionally, there is a calibration function that allows the application of varying pressure values on the sensors and the readout of the resulting voltages. This is done by placing a metal plate over the sensor matrix and applying known force values. Calibration curves for each sensor in the matrix are computed in the process.

\section{Experimental prototype and results}

Since there are currently only 64 sensors, a clearer display is achieved by interpolating the values in the matrix, as shown in Fig. 8 and 9. An alternative display is a 3D surface graph.

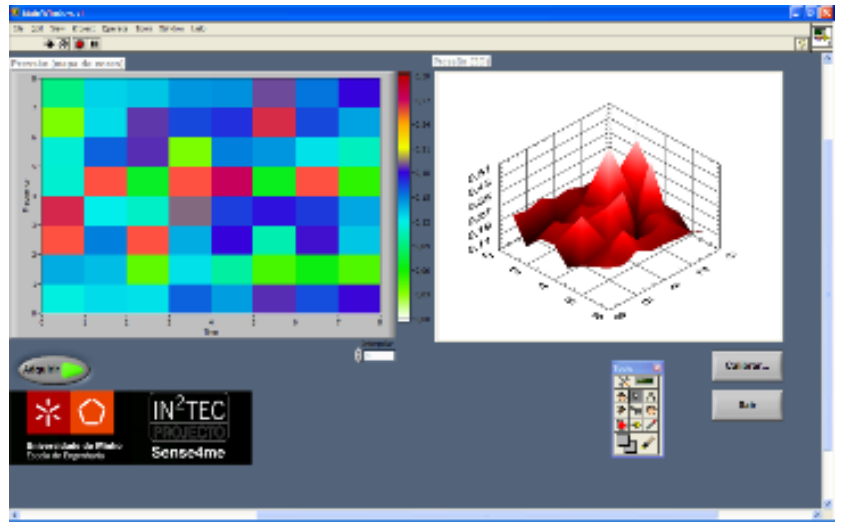

Fig. 8 - Screenshot of the software. Subject is sitting on a wheelchair with sensor matrix. Interpolation disabled in the 2D-colour graph.

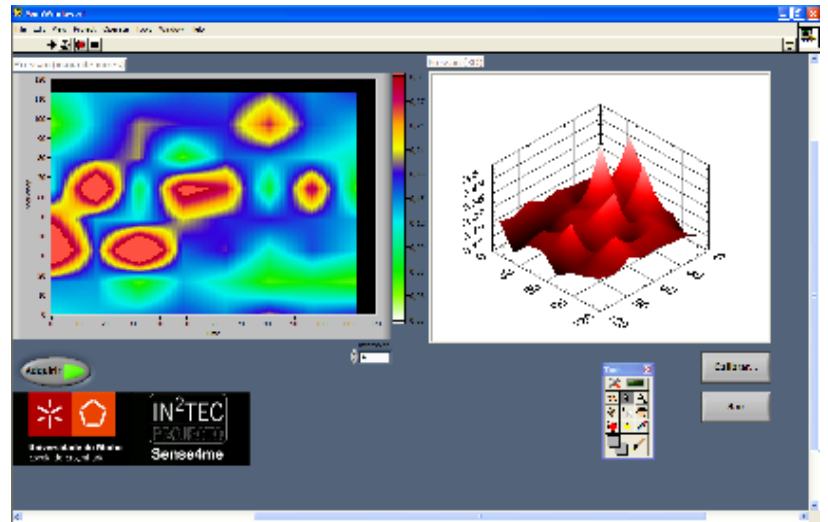

Fig. 9 - Screenshot of the software, same situation, Interpolation enabled.

Fig. 10 shows a picture of the readout electronics board. For this first prototype, this board is composed by 64 channels.

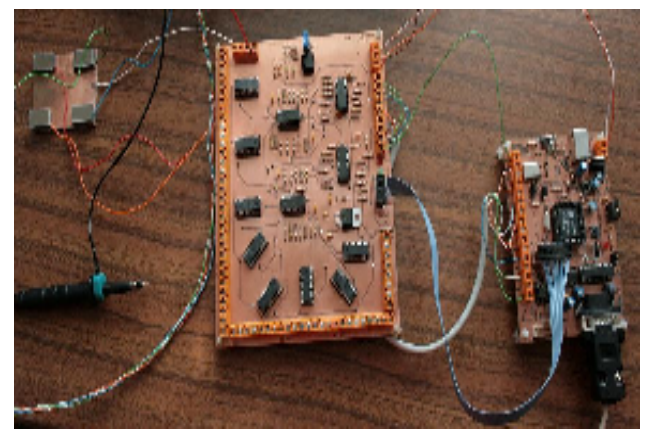

Fig. 10 - Readout electronics board.

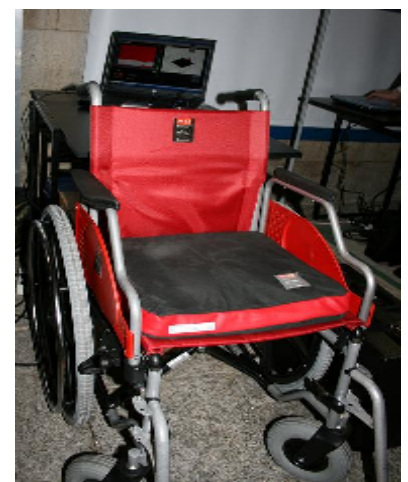

Fig. 11 - First cushion prototype equipped with the sensor matrix and the monitoring system. 


\section{Conclusions}

This paper describes the design, fabrication and experimental results of a pressure sensor array embedded in a cushion and its readout electronics. The pressure sensor array is based on capacitive sensors whose dielectric is constituted by polymeric foam. As this is the first prototype, the obtained results are very promising and encouraging. Nevertheless, there are some issues that must be improved in the next prototypes. The first aspect is the calibration, which should be made easier by allowing the calibration of a single sensor and the use of the resulting calibration parameters for the remaining sensors. The application of a controlled force to the whole sensor array is much more difficult. This can only be achieved with a better control of the dimensional and physical parameters of the sensor fabrication.

Another aspect is the elimination of the rigid copper electrodes. These can cause discomfort to the patient sitting on the wheelchair. In the current prototype, a gel cushion is inserted between the measuring cushion and the patient, but this cushion will somewhat distort the measured force values. Experiments are being conducted to replace the copper plates by textile fabric produced with inserted stainless steel yarns. These metallic yarns will be used as the plates of the capacitive sensors, providing an all-textile, flexible and soft sensor array. The approach has already been validated, a prototype is expected to be demonstrated on short-term.

The use of other type of sensors, namely piezoresistive sensor arrays based on doped hydrogenated nanocrystalline silicon deposited on flexible substrates, temperature and humidity monitoring, protocol of sensor data acquisition versus warnings and actions and the mechanical control are currently under development.

\section{Acknowledgements}

The authors thank the Portuguese Foundation for Science and Technology - FCT (Grant PTDC/SAU-BEB/68678/2006) and School of Engineering from University of Minho (IN ${ }^{2}$ TEC project).

\section{Literature References}

[1] National Pressure Ulcer Advisory Panel. Pressure ulcers: incidence, economics, risk assessment, 1989.

[2] M.H.L. Calibri: A utilização da pesquisa na prática clínica da enfermagem: limites e possibilidades, 2002.

[3] R. Reed: Low serum albumin levels, confusion, and fecal incontinence: are these risk factors for pressure ulcers in mobility impaired hospitalized adults? 2003.

[4] R.A. Allman: Pressure ulcer risk factors among hospitalized patients with activity limitation, 1995.

[5] R.A. Allman: Pressure ulcers among the elderly, 1998.

[6] Fuhrer, M Pressure ulcers in community-resident persons with spinal cord injury: prevalence and risk factors, 1993.

[7] N. Bergstrom, B. Braden: A prospective study of pressure sore risk among institutionalized elderly, 1992.

[8] N. Bergstrom: Treatment of Pressure Ulcers, 1994.

[9] National Institute for Health and Clinical Excellence. Pressure ulcer prevention: pressure ulcer risk assessment and prevention, including the use of pressure-relieving devices (beds, mattresses and overlays) for the prevention of pressure ulcers in primary and secondary care, 2003. 